BMJ Open

Diabetes

Research

\& Care

\section{Risk factors for genital infections in people initiating SGLT2 inhibitors and their impact on discontinuation}

To cite: McGovern AP, Hogg M, Shields BM, et al. Risk factors for genital infections in people initiating SGLT2 inhibitors and their impact on discontinuation. BMJ Open Diab Res Care 2020;8:e001238. doi:10.1136/ bmjdrc-2020-001238

- Additional material is published online only. To view please visit the journal online (http://dx.doi.org/10.1136/ bmjdrc-2020-001238).

Received 29 January 2020 Revised 16 April 2020 Accepted 23 April 2020

Check for updates

(C) Author(s) (or their employer(s)) 2020. Re-use permitted under CC BY. Published by BMJ.

${ }^{1}$ University of Exeter Medical School, Institute of Biomedical and Clinical Science, University of Exeter, Exeter, UK ${ }^{2}$ Institute of Cardiovascular Sciences, University of Glasgow, Glasgow, UK ${ }^{3}$ Radcliffe Department of Medicine, University of Oxford, Oxford, UK

${ }^{4}$ Division of Population Health \& Genomics, School of Medicine, University of Dundee, Dundee, UK

Correspondence to Dr Andrew P McGovern; a.mcgovern@exeter.ac.uk

\section{ABSTRACT}

Introduction To identify risk factors, absolute risk, and impact on treatment discontinuation of genital infections with sodium-glucose co-transporter-2 inhibitors (SGLT2i). Research design and methods We assessed the relationship between baseline characteristics and genital infection in 21004 people with type 2 diabetes initiating SGLT2i and 55471 controls initiating dipeptidyl peptidase-4 inhibitors (DPP4i) in a UK primary care database. We assessed absolute risk of infection in those with key risk factors and the association between early genital infection and treatment discontinuation.

Results Genital infection was substantially more common in those treated with SGLT2i (8.1\% within 1 year) than DPP4i (1.8\%). Key predictors of infection with SGLT2i were female sex (HR 3.64; 95\% Cl 3.23 to 4.11) and history of genital infection; <1 year before initiation (HR 4.38; 3.73 to 5.13 ), $1-5$ years (HR 3.04; 2.64 to 3.51 ), and $>5$ years (HR 1.79; 1.55 to 2.07). Baseline $\mathrm{HbA}_{1 \mathrm{c}}$ was not associated with infection risk for SGLT2i, in contrast to DPP4i where risk increased with higher $\mathrm{HbA}_{1 \mathrm{c}}$. One-year absolute risk of genital infection with SGLT2i was highest for those with a history of prior infection (females $23.7 \%$, males $12.1 \%$ ), compared with those without (females $10.8 \%$, males $2.7 \%$ ). Early genital infection was associated with a similar discontinuation risk for SGLT2i (HR 1.48; 1.21-1.80) and DPP4i (HR 1.58; 1.21-2.07).

Conclusions Female sex and history of prior infection are simple features that can identify subgroups at greatly increased risk of genital infections with SGLT2i therapy. These data can be used to risk-stratify patients. High $\mathrm{HbA}_{1 \mathrm{c}}$ is not a risk factor for genital infections with SGLT2i.

\section{INTRODUCTION}

Sodium-glucose co-transporter-2 inhibitors (SGLT2i) are an increasingly important oral medication class in type 2 diabetes ${ }^{1-3}$ with their use climbing dramatically in recent years. ${ }^{4-7}$ They result in a broadly similar amount of glucose lowering compared with other oral agents but can also reduce blood pressure and result in modest weight loss. ${ }^{8-10}$ In addition to their glucose lowering effects, large-scale clinical trials have demonstrated reduction in cardiovascular and renal outcomes in highrisk groups with type 2 diabetes, ${ }^{11-13}$ as well as

\section{Significance of this study}

What is already known about this subject?

> It has been established that sodium-glucose cotransporter-2 inhibitors (SGLT2i) are associated with greater risk for genital infections. However, patient features which confer the greatest risk are not well elucidated. Female gender is a known risk factor.

What are the new findings?

- Prior history of genital infection and gender are the two main determinants of risk of genital infection with SGLT2i.

- High HbA1c does not increase the risk of genital infection in those starting an SGLT2i, in contrast to those starting a DPP4 inhibitor.

- Genital infections are associated with only a slight increase in treatment discontinuation.

How might these results change the focus of research or clinical practice?

- These data can be used by clinicians to estimate the infection risk for individual patients and hence support more informed decision making.

benefit in patients with heart failure whether or not they have type 2 diabetes. ${ }^{14}$ They can also be used as adjuvant therapy to insulin for the treatment of type 1 diabetes. ${ }^{15}$

SGLT2i reduce hyperglycemia in people with diabetes by increasing urinary excretion of glucose. ${ }^{8} 16$ This induced glycosuria increases the risk of genital infections ${ }^{16}$ and both clinical trials and observational studies demonstrate a 2.5-6-fold increase in genital infections in people using SGLT2i compared with controls. ${ }^{101617}$ A number of factors have been shown to be associated with risk of genital infection in the general population, in particular, female sex and diabetes, especially when glycemic control is poor. ${ }^{18} 19$ However, there has been limited investigation of the risk factors for genital tract infection in those initiating 
SGLT2i therapy, or of the impact of infection on treatment discontinuation outside of a trial setting.

We aimed to determine the factors associated with the risk for developing a genital infection while on SGLT2i treatment and the impact of these infections on treatment discontinuation.

\section{RESEARCH DESIGN AND METHODS}

We conducted a retrospective cohort analysis of people with type 2 diabetes initiating SGLT2i within a large population-based UK cohort; the UK Clinical Practice Research Datalink (CPRD). We examined the prevalence of genital infections during the first year of treatment. We explored the associations between baseline characteristics and history of previous genital infections on infection risk during treatment and examined the impact of genital infections occurring early during treatment on subsequent medication discontinuation. For all analyses, we used people initiating dipeptidyl peptidase- 4 inhibitors (DPP4i) as a comparison cohort. We used all available data up to the point of data extraction, July 2019.

\section{Setting and participants}

CPRD is one of the larger longitudinal population-based medical records datasets in the world and provides a representative sample of the UK population. ${ }^{20}$ People with type 2 diabetes were identified using a method we have previously described in detail. ${ }^{21}$ In summary, they were identified using the presence of a diagnostic code for diabetes and the prescription of one or more glucose lowering medication(s). People were excluded if they had possible type 1 diabetes or another diabetes type. Possible type 1 diabetes was defined as age of diagnosis less than 35 years, treatment with insulin therapy only, or initiation of insulin within 1 year of diagnosis. Other forms of diabetes were excluded using the presence of indicative diagnostic codes including steroid-induced diabetes, gestational diabetes and monogenic diabetes.

The date of diabetes diagnosis was defined as the date of the first of: a diabetes diagnostic code, glucose lowering medication, or glycated hemoglobin $\left(\mathrm{HbA}_{1 \mathrm{c}}\right) \geq 6.5 \% \quad(48$ $\mathrm{mmol} / \mathrm{mol}$ ). Those who had a first diabetes indicator within 3 months of practice registration were excluded as their diagnosis date was uncertain and treatment history could not be determined.

From the cohort of people meeting our criteria for type 2 diabetes with an identifiable date of diagnosis, we included everyone initiating SGLT2i (canagliflozin, dapagliflozin, and empagliflozin). This group then formed our main study cohort. We identified a comparison group of all people initiating DPP4i.

\section{Participant baseline characteristics}

We report the baseline clinical characteristics of both treatment groups at medication initiation; age, sex, duration of diabetes, and body mass index (BMI), $\mathrm{HbA}_{1 c}$, estimated glomerular filtration rate (eGFR), history of genital infections. $\mathrm{BMI}, \mathrm{HbA}_{1 \mathrm{c}}$, and eGFR were defined using the most recent measurement within the 2years prior to the drug start date. We excluded people with missing data for these baseline characteristics or with a baseline eGFR $<45 \mathrm{~mL}$ / $\min$.

\section{Outcomes}

A genital infection was defined as either a diagnosis code specific for a genital infection (eg, candida vaginitis or vulvovaginitis in women, or balanitis, balanoposthitis in men), a prescription for antifungal therapy used specifically to treat genital infections (eg, an antifungal vaginal pessary), or a non-specific diagnosis of "thrush" with a topical antifungal prescribed on the same day. The timing of the most recent infection prior to the start of the index drug was used as a baseline variable and categorized as; occurring within a year, between 1 and 5 years, or longer than 5 years before start of the index drug.

Treatment discontinuation was defined as no repeat prescription issue of the index drug for 3 months (90 days). The discontinuation date was defined as 90 days after the date of the last prescription issue.

\section{Statistical methods}

Associations between baseline characteristics and time to genital infection were assessed using Kaplan-Meier survival plots. We then performed multivariable Coxregression analyses of time to genital infection separately for SGLT2i-treated and DPP4i-treated patients. Multivariable models were defined a priori to include age, sex, duration of diabetes, $\mathrm{HbA}_{1 c}$ prior to treatment, eGFR, BMI, and previous history of genital infections. People with incomplete follow-up were included and censored at loss to follow-up. Time to infection models was also censored at discontinuation of the medication of interest (SGLT2i or DPP4i). Drug additions or switching of background medications was ignored. Complete available follow-up data were used for the Cox models, that is, where follow-up beyond the first year of treatment was available, these data were also included in the models.

As a key baseline variable of interest, we also evaluated the non-linear association between $\mathrm{HbA}_{1 \mathrm{c}}$ prior to drug initiation and subsequent risk for genital infection by fitting continuous baseline $\mathrm{HbA}_{1 \mathrm{c}}$ as a restricted cubic spline with three knots, with adjustment for the same factors used in the multivariable models.

Based on the results of Cox regression models, we defined four important clinical risk groups using the two most discriminative baseline features: sex, and history of one or more genital infection. For each risk group, we report the proportion of people developing a genital infection during the first year of treatment. We also report the annual cumulative incidence of infection out to 4 years, in each risk group.

The impact of early infection on discontinuation was assessed using multivariable Cox-regression separately for SGLT2i-treated and DPP4i-treated patients. Multivariable models were adjusted for the same baseline variables included in the infection risk models: age, gender, 
duration of diabetes, $\mathrm{HbA}_{1 \mathrm{c}}$ prior to treatment, eGFR, BMI, and previous history of genital infections.

\section{Sensitivity analyses}

Patient characteristics influence treatment selection and therefore potentially influence the results of our primary analysis. To explore this possibility further, we repeated the primary analysis in propensity score-matched subgroups. Matching by treatment choice (SGLT2i or DPP4i) was performed using a 1:1 matching ratio and a nearest neighbor matching algorithm (R MatchIt V3.0.2). Variables included in the propensity matching were: age, sex, duration of diabetes, number of concurrent medications, $\mathrm{HbA}_{1 c}$ prior to treatment, eGFR, BMI, and previous history of genital infections. We compared the baseline characteristics between the SGLT2i and DPP4i matched groups using the $\chi^{2}$ test for categorical variables and the unpaired t-test for continuous data. All reported $\mathrm{p}$ values are two-sided. We used these matched cohorts to replicate the primary analysis for genital infection risk. We also used them to examine the impact of treatment selection on genital infection rates overall and in our four clinical risk groups, that is, we compare the additional risk for infection incurred by treatment with an SGLT2i compared with a DPP4i.

Posthoc, we also performed two additional analysis. The first, a sensitivity analysis, explored the impact of removing genital infections identified using a combination of the non-specific diagnosis code "thrush" and a simultaneous topical treatment. This sensitivity analysis was only performed in females, as there was no condition specific treatment available (eg, antifungal pessaries) for males and as there were fewer diagnosis specific codes in males compared with females. The second explored associations between concurrent medications with potential associations with fungal infection: corticosteroids, ${ }^{22}$ oestrogen therapies, ${ }^{23} 24$ and non-steroid immune-modulating medications .$^{25}$ These were defined as being prescribed at baseline if a prescription was present in the clinical record within the 3 months prior to initiation of the index drug. All oral corticosteroids were included for analysis; topical or other preparations were not considered. Oestrogen therapies comprised hormone replacement therapies, combined oral contraceptives, and topical vaginal oestrogen preparations. Immune-modulating medications comprised all nonsteroid immunosuppressants listed in the British National Formulary including thiopurines (azathioprine and mercaptopurine), methotrexate, calcineurin inhibitors (tacrolimus and cyclosporine) and monoclonal antibodies.

\section{RESULTS}

Within CPRD, we identified 302857 people who met our criteria for type 2 diabetes. Of these, 22928 were initiated on an SGLT2i and 67104 on a DPP4i. There was incomplete baseline data on $\mathrm{HbA}_{1 \mathrm{c}}$, eGFR, or BMI for 1920 (8.4\%) people initiating an SGLT2i and 11623 (17.3\%) initiating a DPP4i. There were some baseline differences between the remaining cohorts; those initiating SGLT2is
Table 1 The baseline characteristics of people initiated on an SGLT2i or DPP4i

\begin{tabular}{|c|c|c|}
\hline & $\begin{array}{l}\text { SGLT2i } \\
(n=21008)\end{array}$ & $\begin{array}{l}\text { DPP4i } \\
(n=55481)\end{array}$ \\
\hline Age (years) & $60.4(9.3)$ & $63.3(10.8)$ \\
\hline Female (n (\%)) & $8115(38.6)$ & $22274(40.1)$ \\
\hline Duration of diabetes (years) & $9.1(5.4)$ & $7.9(5.4)$ \\
\hline$<5$ years $(\mathrm{n}(\%))$ & $6089(29.0)$ & 21003 (37.9) \\
\hline $5-10$ years $(n(\%))$ & $7296(34.7)$ & $19414(35.0)$ \\
\hline$>10$ years $(\mathrm{n}(\%))$ & 7623 (36.3) & $15064(27.2)$ \\
\hline $\begin{array}{l}\text { Number of concurrent } \\
\text { diabetes medications }\end{array}$ & $1.7(0.8)$ & $1.4(0.7)$ \\
\hline $\mathrm{HbA} 1 \mathrm{c}(\%(\mathrm{mmol} / \mathrm{mol}))$ & $78.2(16.7)$ & $73.1(16.6)$ \\
\hline$<8.0(<64)(\mathrm{n}(\%))$ & $4401(20.9)$ & $19067(34.4)$ \\
\hline $8.0-9.5(64-80)(n(\%))$ & $8547(40.7)$ & $21665(39.0)$ \\
\hline$>9.5(>80)(n(\%))$ & $8060(38.4)$ & $14749(26.6)$ \\
\hline eGFR (mL/min) & $87.9(15.2)$ & $82.7(17.5)$ \\
\hline $45-<60 \mathrm{~mL} / \min (\mathrm{n}(\%))$ & $890(4.2)$ & $6997(12.6)$ \\
\hline 60-90 (n (\%)) & $9674(46.0)$ & $27245(49.1)$ \\
\hline$>90(\mathrm{n}(\%))$ & $10444(49.7)$ & $21239(38.3)$ \\
\hline $\mathrm{BMI}\left(\mathrm{kg} / \mathrm{m}^{2}\right)$ & $34.4(6.7)$ & $32.7(6.6)$ \\
\hline$<25 \mathrm{~kg} / \mathrm{m}^{2}(\mathrm{n}(\%))$ & $946(4.5)$ & $5156(9.3)$ \\
\hline $25-30 \mathrm{~kg} / \mathrm{m}^{2}$ (n (\%)) & $4747(22.6)$ & $16040(28.9)$ \\
\hline$>30 \mathrm{~kg} / \mathrm{m}^{2}$ (n (\%)) & $15311(72.9)$ & $34275(61.8)$ \\
\hline \multicolumn{3}{|c|}{ Previous genital mycosis (n (\%)) } \\
\hline Never & $16877(80.3)$ & $46663(84.1)$ \\
\hline$<1$ year & $722(3.4)$ & $1753(3.2)$ \\
\hline $1-5$ years & $1363(6.5)$ & $2943(5.3)$ \\
\hline$>5$ years & $2046(9.7)$ & $4122(7.4)$ \\
\hline Corticosteroid use (n (\%)) & $2317(4.2)$ & $718(3.4)$ \\
\hline Oestrogen use (n (\%)) & $665(1.2)$ & $252(1.2)$ \\
\hline $\begin{array}{l}\text { Immunomodulator use (n } \\
(\%) \text { ) }\end{array}$ & $629(1.1)$ & $219(1.0)$ \\
\hline
\end{tabular}

All values shown are mean (SD) unless otherwise stated. BMI, body mass index; DPP4i, Dipeptidyl peptidase-4 inhibitor; eGFR, estimated glomerular filtration rate; SGTL2, sodium-glucose co-transporter-2.

were younger, had a higher male proportion, longer duration of diabetes, higher $\mathrm{HbA}_{1 \mathrm{c}}$, eGFR, and BMI, and more previous genital infections (table 1). The SGLT2i and DPP4i most commonly initiated were dapagliflozin and sitagliptin, respectively (online supplementary table S1).

\section{Women and those with prior infection have higher genital infection risk}

Genital infections were more common within the first year of treatment in those on SGLT2i $(8.1 \%$; $95 \% \mathrm{CI}$ $7.6 \%$ to $8.5 \%)$ compared with DPP4i $(1.8 \%$; $95 \%$ CI $1.7 \%$ to $1.9 \%$ ). Females were more likely to have a genital infection than males (figure 1A). Those with a history of 
A

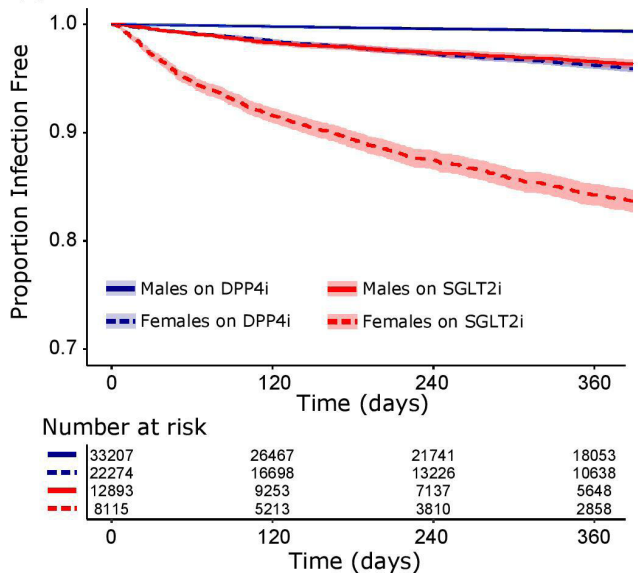

B

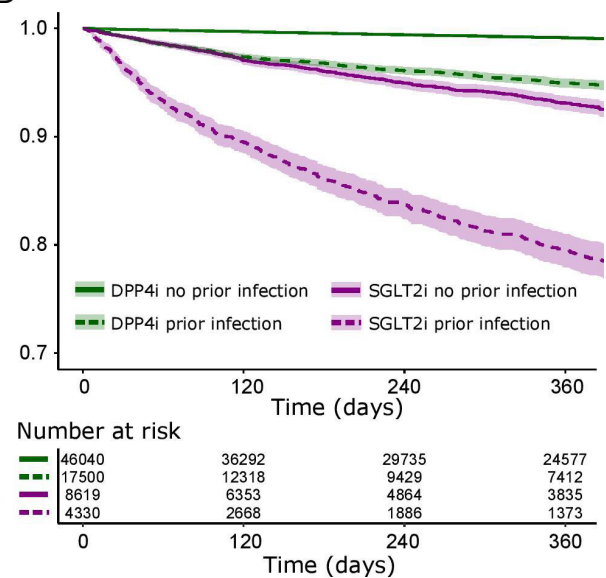

Figure 1 The proportion of people free from genital infection since drug initiation (A) by sex and medication class (SGLT2i and DPP4i) and (B) by history of prior genital infection and medication class. Number of events by group: (A) DPP4 inhibitor males $n=228$, DPP4 inhibitor females $n=914$, SGLT2i males $n=371$, SGLT2i females $n=1092$, (B) DPP4 inhibitor no prior infection $n=484$, DPP4 inhibitor and prior infection $n=658$, SGLT2i no prior infection $n=729$, SGLT2i and prior infection $n=734$. The shaded area represents the $95 \% \mathrm{Cl}$. DPP4i, dipeptidyl peptidase-4 inhibitor; SGTL2i, sodium-glucoseco-transporter-2 inhibitor.

previous genital infection were more likely to experience another infection after treatment initiation (figure 1B). High $\mathrm{HbA}_{1 \mathrm{c}}$ is not an additional risk factor for genital infection with SGLT2is

In multivariable models, female gender, higher BMI, and genital infection were independently associated with risk for future genital infection in people treated with both
SGLT2is and DPP4is (table 2). The more recent the previous genital infection, the greater the subsequent infection risk. Higher $\mathrm{HbA}_{1 \mathrm{c}}$ was associated with greater infection risk in people using DPP4is but not SGLT2is (table 2 and figure 2). Sensitivity analysis using matched subgroups demonstrated the same pattern of association (online supplementary tables S2 and S3), as did analysis

Table 2 Associations between patient characteristics at medication initiation and subsequent genital infections with SGLT2 and DPP4 inhibitors

\begin{tabular}{|c|c|c|}
\hline & $\begin{array}{l}\text { SGLT2i } \\
(\mathrm{n}=21008) \\
\text { HR }(95 \% \mathrm{Cl}) \mathrm{P} \text { value }\end{array}$ & $\begin{array}{l}\text { DPP4i } \\
(\mathrm{n}=55481) \\
\text { HR }(95 \% \mathrm{Cl}) \mathrm{P} \text { value }\end{array}$ \\
\hline Age (years) & $0.99(0.99$ to 1.00$) p=0.069$ & 0.98 (0.98 to 0.99$) p<0.001$ \\
\hline Female & 3.66 (3.24 to 4.13$) p<0.001$ & 4.05 (3.51 to 4.66$) p<0.001$ \\
\hline \multicolumn{3}{|c|}{ Duration of diabetes (Reference group: $<5$ years) } \\
\hline $5-10$ years & 0.87 (0.77 to 0.98$) p=0.024$ & $1.01(0.89$ to 1.14$) p=0.91$ \\
\hline$>10$ years & $0.93(0.81$ to 1.06$) p=0.24$ & 1.05 (0.91 to 1.22$) p=0.49$ \\
\hline \multicolumn{3}{|c|}{ HbA1c (Reference group:<8.0\% (<64 mmol/mol)) } \\
\hline $8.0 \%-9.5 \%(64-80 \mathrm{mmol} / \mathrm{mol})$ & $1.11(0.97$ to 1.27$) p=0.13$ & $1.12(0.98$ to 1.27$) \mathrm{p}=0.108$ \\
\hline$>9.5 \%(>80 \mathrm{mmol} / \mathrm{mol})$ & 0.95 (0.83 to 1.09$) \mathrm{p}=0.49$ & $1.48(1.29$ to 1.71$) p<0.001$ \\
\hline \multicolumn{3}{|l|}{ eGFR (Reference group:>90 mL/min) } \\
\hline $60-90 \mathrm{~mL} / \mathrm{min}$ & $0.78(0.56$ to 1.08$) p=0.13$ & $0.76(0.61$ to 0.96$) p=0.019$ \\
\hline$<60 \mathrm{~mL} / \mathrm{min}$ & $1.01(0.90$ to 1.13$) p=0.87$ & $0.96(0.85$ to 1.09$) \mathrm{p}=0.57$ \\
\hline \multicolumn{3}{|l|}{ BMI (Reference group: $25-30$ kg/m²) } \\
\hline$<25 \mathrm{~kg} / \mathrm{m}^{2}$ & $0.78(0.55$ to 1.10$) p=0.15$ & $0.70(0.53$ to 0.93$) p=0.016$ \\
\hline$>30 \mathrm{~kg} / \mathrm{m}^{2}$ & $1.22(1.07$ to 1.39$) \mathrm{p}=0.005$ & 1.29 (1.13 to 1.48$) p<0.001$ \\
\hline \multicolumn{3}{|c|}{ Previous genital mycosis (Reference group: no previous infection) } \\
\hline$<1$ year & 4.47 (3.81 to 5.24$) p<0.001$ & $8.90(7.67$ to 10.34$) p<0.001$ \\
\hline $1-5$ years & 3.04 (2.64 to 3.51$) p<0.001$ & 4.13 (3.54 to 4.82$) p<0.001$ \\
\hline$>5$ years & 1.77 (1.53 to 2.04$) \mathrm{p}<0.001$ & 2.00 (1.68 to 2.38$) p<0.001$ \\
\hline C-statistic & 0.76 & 0.82 \\
\hline
\end{tabular}

BMI, body mass index; DDP4i, dipeptidyl peptidase-4 inhibitor; eGFR, estimated glomerular filtration rate; HR, hazard ratio; SGTL2, sodium-glucose co-transporter-2. 

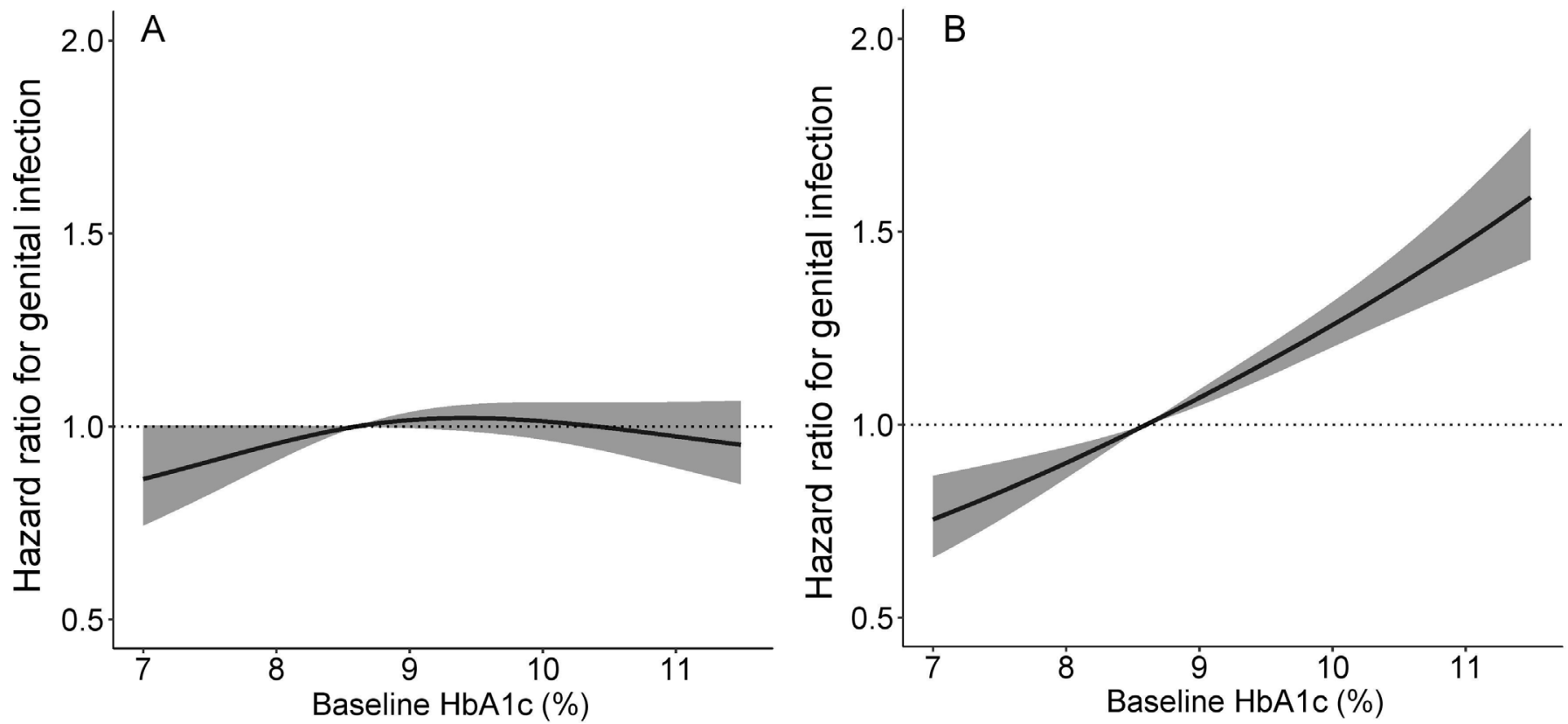

Figure 2 The association between $\mathrm{HbA} 1 \mathrm{c}$ prior to medication initiation and genital infection after initiation of (A) an SGLT2 inhibitor and (B) a DPP4 inhibitor. Analyses are adjusted for age, gender, duration of diabetes, eGFR, BMI, and prior history of infection. The shaded area represents the $95 \% \mathrm{Cl}$. A median $\mathrm{HbA} 1 \mathrm{c}$ value of $8.6 \%(71 \mathrm{mmol} / \mathrm{mol})$ is used as the reference point. BMI, body mass index; DPP4i, dipeptidyl peptidase-4 inhibitor; eGFR, estimated glomerular filtration rate; SGTL2, sodiumglucoseco-transporter-2.

excluding those with non-specific diagnosis codes (online supplementary table $\mathrm{S} 4$ ).

Including the use of oestrogens, corticosteroids, and other immune-modulating medications did not significantly modify the associations identified (online supplementary table S5). The use of these medications at treatment initiation was not associated with significantly increased risk for infection in the SGLT2i group but corticosteroid and oestrogen use was associated with increased risk in those initiating DPP4is.

\section{Future genital infection risk can be stratified using gender and infection history}

Using the results of the regression models, we defined four key risk groups: males without a history of genital infection, males with a history of genital infection, females without a history of genital infection, females with a history of genital infection. These groups can be used to estimate the absolute risk for genital infection over the first year of treatment with an SGLT2i compared

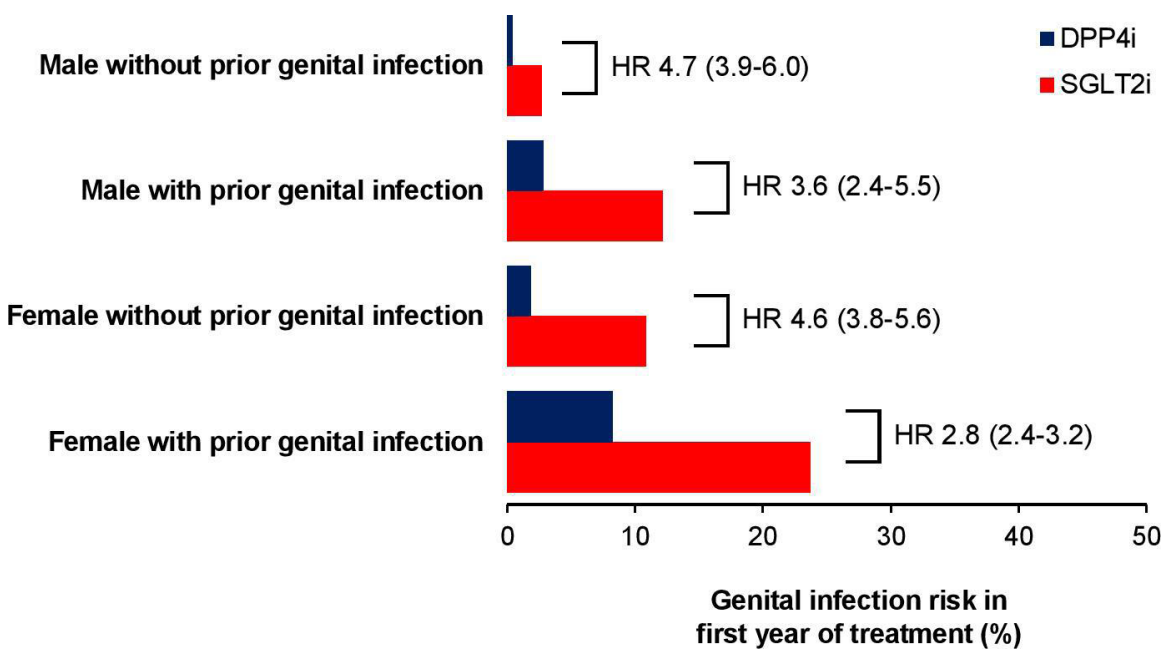

Figure 3 The absolute and relative risk of having a genital infection with an SGLT2i versus DPP4i by risk group. HRs are derived from propensity matched cohorts (online supplementary table S2) and are adjusted for age, gender, duration of diabetes, $\mathrm{HbA}_{1 \mathrm{c}}$, eGFR, BMI, and prior history of infection (online supplementary table S8). Numbers in brackets are $95 \%$ Cls. BMI, body mass index; DPP4i, dipeptidyl peptidase-4 inhibitor; eGFR, estimated glomerular filtration rate; HR, hazard ratio; SGTL2i, sodium-glucoseco-transporter-2 inhibitor. 
with a DPP4i (figure 3 and online supplementary table S6) and over longer durations (online supplementary table S7) and the HR for infection associated with using an SGLT2i compared with a DPP4i (figure 3 and online supplementary table $\mathrm{S} 8$ ).

\section{Genital infections are associated with a greater risk of discontinuation}

Genital infections occurring within the first month of treatment were associated with a greater adjusted risk for subsequent discontinuation with both SGLT2i ( $19.4 \%$ without discontinued versus $31.8 \%$ with by 1 year; adjusted HR 1.48; 95\% CI 1.21 to 1.81 ) and DPP4i (19.0\% without versus $36.4 \%$ with by 1 year; adjusted HR 1.58 ; $95 \%$ CI 1.21 to 2.07$)$.

\section{CONCLUSION}

Our study shows sex and history of genital infection can be used to stratify the risk of genital infections over the first year of SGLT2i treatment. Absolute risk is markedly higher in those with prior infection (females 23.7\%, males $12.1 \%$ ), compared with those without (females $10.8 \%$, males $2.7 \%$ ). In contrast, we found no evidence of an association between high $\mathrm{HbA}_{1 \mathrm{c}}$ levels and increased risk of infection with SGLT2i, though we did for DPP4i. Early genital infection was associated with an increased discontinuation risk with SGLT2is (HR 1.48; 1.21 to 1.80 ); this effect was also observed with DPP4is (HR 1.58; 1.2 to 2.1$)$.

\section{Comparison with the literature}

Our findings suggest that the prevalence of genital infections in clinical practice in people using SGLT2is is slightly higher overall $(8.1 \%$; $95 \%$ CI $7.6 \%$ to $8.5 \%)$ that that reported in large clinical trials with SGLT2is $2.5 \%$ to $6.5 \%) .{ }^{11-13}$ Despite the lower incidence in trials, metaanalyses of trial data show a 4-6-fold increased risk of genital infection with SGLT2i compared with placebo, consistent with our findings. ${ }^{10} 26$ Prior history of genital infection was also associated with a greater risk of further infection on SGLT2i treatment in clinical trials. ${ }^{16}$ This finding fits clinical experience.

The associations we identified between sex and prior history of infection are supported in a much smaller national audit of SGLT2i use from secondary care; ${ }^{27}$ however, multivariable analysis was not performed in this study. A US claims-based study also found similar sex associations. ${ }^{28}$ Our data provide the first estimates of absolute and relative genital infection risk with SGLT2i across clinical groups defined using simple patient characteristics. To our knowledge, no previous study has explored the independent association between HbAlc and genital infection with SGLT2i, analyzed over a wide range of $\mathrm{HbA}_{1 \mathrm{c}}$ values and this is a critical and novel finding. We also provide the first simple clinical stratification tool to estimate genital infection risk across four key risk groups.

\section{Strengths and limitations}

Important strengths of our analysis are the large cohort size, high levels of data completeness in the diabetes cohort from which our sample was derived and the realworld nature of the data. These factors mean our results have broad generalizability and can be applied to current clinical practice. There are some noteworthy limitations. Our analysis relies, in part, on correct clinical coding of genital infections and is therefore subject to inaccuracies resulting from non-coding or miscoding of genital infections. However, the rates of genital infections in our analysis are consistent with other smaller studies which used case note review to identify genital infections and therefore not subject to coding errors. ${ }^{29}{ }^{30}$ Topical antifungal treatments are available to purchase 'over-the-counter' and therefore some people may have treated their genital infections without presenting to primary care; we therefore may have systematically under recorded infection rates. The severity of infections was not available; for example, paraphimosis and other complication data were not sufficiently well recorded to analyze these outcomes. An additional limitation is that data on the reasons for treatment discontinuation were not available for this study.

\section{Clinical implications}

We provide the first simple clinical method for stratifying risk for genital infections with SGLT2i across four key risk groups; both absolute risks and HRs compared with DPP4i. This can be used in clinical practice to support patients to make more informed treatment selection decisions. If those at highest risk elect to start an SGLT2i, then practitioners should pay particular attention to counseling regarding genital hygiene and when to start antifungal treatments. While early infection was associated with a greater chance of discontinuation, this effect was also seen with DPP4i. Other recent work has demonstrated that simple clinical features can be used to stratify risk of side-effects with sulfonylurea and thiazolidinedione treatment. ${ }^{31} 32$ Along this, our findings provide a starting point for a more personalized approach to selecting type 2 diabetes treatment after metformin, with specific medication targeted according to individuals or subgroups based on their biological or risk characteristics. ${ }^{33}$

Interestingly, we found that people with high $\mathrm{HbA}_{1 \mathrm{c}}$ are not at additional genital infection risk with SGLT2i. This may be because glycosuria is a universal feature of SGLT2i treatment, occurring even in people with a normal $\mathrm{HbA}_{1 c}{ }^{34}$ It is therefore plausible that the association between glycosuria and genital infection reaches a saturation point above which increased glycosuria does not increase infection risk. This threshold may be exceeded with SGLTi use even in those with a relatively low $\mathrm{HbA}_{1 c}$. By contrast, in those on DPP4i treatment, the amount of glycosuria will be low in those with the lowest serum glucose and will progressively increase with higher HbAlc as the serum glucose progresses past the renal threshold of maximum glucose resorption. ${ }^{35}$ It is similarly interesting that corticosteroids, immune-modulating medications, and oestrogen therapies 
were not associated with significant additional infection risk in those using SGLT2i. The reasons for this are unclear. While we are likely to be underpowered (particularly with immune-modulating therapies) to detect small increases in risk, our data reassuringly suggest no substantial increase in risk if these treatments are used concurrently with SGLT2i.

In conclusion, genital infections are common in people on SGLT2i in clinical practice. Major risk factors of infection are female sex and prior infection but not baseline $\mathrm{HbA}_{1 c}$, meaning risk can be stratified by these two simple clinical features.

Acknowledgements The authors thank the staff of the NIHR Exeter Clinical Research Facility and NIHR Diabetes Research Network for assistance with conducting the study.

Collaborators Additional collaborators in the MASTERMIND consortium: William E Henley; Mike Lonergan; Lauren R Rodgers; Willie T Hamilton; Catherine Angwin; Kennedy J Cruickshank; Andrew J Farmer; Stephen C L Gough; Alastair M Gray; Christopher Hyde; Christopher Jennison; Mark Walker.

Contributors APM, JMD, and AGJ designed the study and drafted the article. BMS and JMD extracted and processed the data. APM and MH analyzed the data. BMS, NAS, RRH, ERP, and ATH contributed to the study design, provided support for the analysis and interpretation of the results. All authors critically revised the article and approved the final version.

Funding The MASTERMIND (MRC APBI Stratification and Extreme Response Mechanism IN Diabetes) consortium is funded by the UK Medical Research Council study grant number MR/N00633X/1. BMS and ATH are supported by the NIHR Exeter Clinical Research Facility. ATH is an NIHR Senior Investigator. ATH is a Wellcome Trust Senior Investigator. AGJ is supported by an NIHR Clinician Scientist award (17/0005624). APM is an NIHR academic clinical fellow. ERP is a Wellcome Trust New Investigator (102820/Z/13/Z). JMD is supported by an Independent Fellowship funded by Research England's Expanding Excellence in England (E3) fund. RRH is an emeritus UK National Institute for Health Research Senior Investigator.

Competing interests APM declares research support from Eli Lilly and Company, Pfizer, and AstraZeneca. NAS has consulted or been on speakers bureaus for Amgen, Astrazeneca, Boehringer Ingelheim, Janssen, Eli-Lilly, Novo Nordisk, NAPP pharmaceuticals, Pfizer, and Sanofi and received grant support from Boehringer Ingelheim. ERP declares personal fees from Eli Lilly and Company, Novo Nordisk, and AstraZeneca. RRH reports research support from AstraZeneca, Bayer and Merck Sharp \& Dohme, and personal fees from Bayer, Intarcia, Merck Sharp \& Dohme, Novartis, and Novo Nordisk. AGJ is the guarantor of this work and, as such, had full access to all the data in the study and takes responsibility for the integrity of the data and the accuracy of the data analysis.

Patient consent for publication Not required.

Ethics approval Scientific approval was granted by the CPRD Independent Scientific Advisory Committee (ISAC 13_177R). CPRD has ethical approval from the UK National Research Ethics Service Committee (NRES). No patient identifiable information was available to researchers.

Provenance and peer review Not commissioned; externally peer reviewed.

Data availability statement Data may be obtained from a third party and are not publicly available. Data used for this study are those collated as part of the Clinical Practice Research Datalink (CPRD). Information on access to this dataset can be found at https://www.cprd.com/Data-access.

Open access This is an open access article distributed in accordance with the Creative Commons Attribution 4.0 Unported (CC BY 4.0) license, which permits others to copy, redistribute, remix, transform and build upon this work for any purpose, provided the original work is properly cited, a link to the licence is given, and indication of whether changes were made. See: https://creativecommons.org/ licenses/by/4.0/.

\section{ORCID iDs}

Andrew P McGovern http://orcid.org/0000-0002-6833-9399

Beverley M Shields http://orcid.org/0000-0003-3785-327X

Rury R Holman http://orcid.org/0000-0002-1256-874X

\section{REFERENCES}

1 Authors/Task Force Members, Rydén L, Grant PJ, et al. Esc guidelines on diabetes, pre-diabetes, and cardiovascular diseases developed in collaboration with the EASD: the task force on diabetes, pre-diabetes, and cardiovascular diseases of the European Society of cardiology (ESC) and developed in collaboration with the European association for the study of diabetes (EASD). Eur Heart $J$ 2013;34:3035-87.

2 Davies MJ, D'Alessio DA, Fradkin J, et al. Management of hyperglycemia in type 2 diabetes, 2018. A consensus report by the American diabetes association (ADA) and the European association for the study of diabetes (EASD). Diabetes Care 2018;41:2669-701.

3 National Institute for Health and Care Excellence. NICE guideline [NG28] - Type 2 diabetes in adults: management, 2019. Available: https://www.nice.org.uk/guidance/ng28

4 Dennis JM, Henley WE, McGovern AP, et al. Time trends in prescribing of type 2 diabetes drugs, glycaemic response and risk factors: a retrospective analysis of primary care data, 2010-2017. Diabetes Obes Metab 2019;21:1576-84.

5 Curtis HJ, Dennis JM, Shields BM, et al. Time trends and geographical variation in prescribing of drugs for diabetes in England from 1998 to 2017. Diabetes Obes Metab 2018;20:2159-68.

6 Persson F, Bodegard J, Lahtela JT, et al. Different patterns of second-line treatment in type 2 diabetes after metformin monotherapy in Denmark, Finland, Norway and Sweden (D360 Nordic): a multinational observational study. Endocrinol Diabetes Metab 2018;1:e00036.

7 Montvida O, Shaw J, Atherton JJ, et al. Long-Term trends in Antidiabetes drug usage in the U.S.: real-world evidence in patients newly diagnosed with type 2 diabetes. Diabetes Care 2018;41:69-78.

8 Brown E, Rajeev SP, Cuthbertson DJ, et al. A review of the mechanism of action, metabolic profile and haemodynamic effects of sodium-glucose co-transporter-2 inhibitors. Diabetes Obes Metab 2019;21:9-18.

9 Storgaard H, Gluud LL, Bennett C, et al. Benefits and harms of sodium-glucose co-transporter 2 inhibitors in patients with type 2 diabetes: a systematic review and meta-analysis. PLoS One 2016;11:e0166125.

10 Zaccardi F, Webb DR, Htike ZZ, et al. Efficacy and safety of sodiumglucose co-transporter-2 inhibitors in type 2 diabetes mellitus: systematic review and network meta-analysis. Diabetes Obes Metab 2016;18:783-94.

11 Zinman B, Wanner C, Lachin JM, et al. Empagliflozin, cardiovascular outcomes, and mortality in type 2 diabetes. N Engl $\mathrm{J}$ Med 2015;373:2117-28.

12 Neal B, Perkovic V, Mahaffey KW, et al. Canagliflozin and cardiovascular and renal events in type 2 diabetes. N Engl J Med 2017;377:644-57.

13 Wiviott SD, Raz I, Bonaca MP, et al. Dapagliflozin and cardiovascular outcomes in type 2 diabetes. N Engl J Med 2019;380:347-57.

14 McMurray JJV, Solomon SD, Inzucchi SE, et al. Dapagliflozin in patients with heart failure and reduced ejection fraction. $N$ Engl $J$ Med 2019;381:1995-2008.

15 Wolfsdorf JI, Ratner RE. Sglt inhibitors for type 1 diabetes: proceed with extreme caution. Diabetes Care 2019;42:991-3.

16 Geerlings S, Fonseca V, Castro-Diaz D, et al. Genital and urinary tract infections in diabetes: impact of pharmacologically-induced glucosuria. Diabetes Res Clin Pract 2014;103:373-81.

17 Lega IC, Bronskill SE, Campitelli MA, et al. Sodium glucose cotransporter 2 inhibitors and risk of genital mycotic and urinary tract infection: a population-based study of older women and men with diabetes. Diabetes Obes Metab 2019;21:2394-404.

18 Sobel JD. Vulvovaginal candidosis. Lancet 2007;369:1961-71.

19 Hine JL, de Lusignan S, Burleigh D, et al. Association between glycaemic control and common infections in people with type 2 diabetes: a cohort study. Diabet Med 2017;34:551-7.

20 Herrett E, Gallagher AM, Bhaskaran K, et al. Data resource profile: clinical practice research Datalink (CPRD). Int J Epidemiol 2015;44:827-36.

21 Rodgers LR, Weedon MN, Henley WE, et al. Cohort profile for the mastermind study: using the clinical practice research Datalink (CPRD) to investigate stratification of response to treatment in patients with type 2 diabetes. BMJ Open 2017;7:e017989.

22 Farhan MA, Moharram AM, Salah T, et al. Types of yeasts that cause vulvovaginal candidiasis in chronic users of corticosteroids. Med Mycol 2019;57:681-7.

23 Spinillo A, Capuzzo E, Nicola S, et al. The impact of oral contraception on vulvovaginal candidiasis. Contraception 1995;51:293-7. 
24 Spinillo A, Bernuzzi AM, Cevini C, et al. The relationship of bacterial vaginosis, Candida and Trichomonas infection to symptomatic vaginitis in postmenopausal women attending a vaginitis clinic. Maturitas 1997;27:253-60.

25 Orlicka K, Barnes E, Culver EL. Prevention of infection caused by immunosuppressive drugs in gastroenterology. Ther Adv Chronic Dis 2013:4:167-85.

26 Wu JHY, Foote C, Blomster J, et al. Effects of sodium-glucose cotransporter-2 inhibitors on cardiovascular events, death, and major safety outcomes in adults with type 2 diabetes: a systematic review and meta-analysis. Lancet Diabetes Endocrinol 2016;4:411-9.

27 Johnsson KM, Ptaszynska A, Schmitz B, et al. Vulvovaginitis and balanitis in patients with diabetes treated with dapagliflozin. $J$ Diabetes Complications 2013;27:479-84.

28 Dave CV, Schneeweiss S, Patorno E. Comparative risk of genital infections associated with sodium-glucose co-transporter-2 inhibitors. Diabetes Obes Metab 2019;21:434-8.

29 Mirabelli M, Chiefari E, Caroleo P, et al. Long-Term effectiveness and safety of SGLT-2 inhibitors in an Italian cohort of patients with type 2 diabetes mellitus. J Diabetes Res 2019;2019:1-8.
30 McGovern AP, Dutta N, Munro N, et al. Dapagliflozin: clinical practice compared with pre-registration trial data. $\mathrm{Br} J$ Diabetes 2014;14:138-43.

31 Dennis JM, Henley WE, Weedon MN, et al. Sex and BMI alter the benefits and risks of sulfonylureas and thiazolidinediones in type 2 diabetes: a framework for evaluating stratification using routine clinical and individual trial data. Diabetes Care 2018;41:1844-53.

32 Dennis JM, Shields BM, Jones AG, et al. Evaluating associations between the benefits and risks of drug therapy in type 2 diabetes: a joint modeling approach. Clin Epidemiol 2018;10:1869-77.

33 Hattersley AT, Patel KA. Precision diabetes: learning from monogenic diabetes. Diabetologia 2017;60:769-77.

34 Komoroski B, Vachharajani N, Boulton D, et al. Dapagliflozin, a novel SGLT2 inhibitor, induces dose-dependent glucosuria in healthy subjects. Clin Pharmacol Ther 2009;85:520-6.

35 Farber SJ, Berger EY, Earle DP. Effect of diabetes and insulin of the maximum capacity of the renal tubules to reabsorb glucose. J Clin Invest 1951;30:125-9. 